\title{
Parameters affecting the transient response of an impacting beam
}

\author{
Weiping $\mathrm{Xu}^{\mathrm{a}}$ and Elizabeth K. Ervin ${ }^{\mathrm{b}, *}$ \\ ${ }^{a}$ Key Laboratory of Transportation Tunnel Engineering, Ministry of Education, School of Civil Engineering, \\ Southwest Jiaotong University, Chengdu, SiChuan, China \\ ${ }^{\mathrm{b}}$ Civil Engineering, The University of Mississippi, Oxford, MS, USA
}

Received 14 December 2012

Revised 13 March 2013

Accepted 25 March 2013

\begin{abstract}
Impact causes shock waves that may be unexpected and damaging. A computationally efficient impact model with a generic beam which is discrete in time and continuous in space was undertaken; an Euler-Bernoulli beam with adjustable boundary conditions and variable contact location is numerically studied under a pulse loading. Experiments on a cantilever beam were carried out to verify the effects of influential parameters. A half-sine pulse excitation was applied through a mechanical shaker, and the deflection was captured by a high speed camera. Numerous test cases were conducted that varied pulse duration, pulse amplitude, and clearance. Decreasing the pulse duration lowers all deflection amplitudes, but the time in contact is insensitive. No gap causes minimal beam response, and increasing gap generates greater deflection. Representative test cases were selected for validating the theoretical model. When comparing numerical simulation with experimental results, satisfactory agreement for amplitude and duration can be reached even with raw input parameters. The contribution of this study is the incorporation of unique pulse loading, changeable boundary conditions, adjustable contact/impact situations, comprehensive parameter studies, and high speed photography.
\end{abstract}

Keywords: Impact, contact, experimental validation, continuous structure

\section{Introduction}

Machinery can routinely experience internal contact. For example, the wear of steam generator tubes and nuclear reactor control rods has great significance. In a heat exchanger system, baffle plates are used to limit the deflection of tubes under flow-induced or transient loading. Impacts can occur between the long slender tubes and the stiff baffle plates. A leak in any of the tubes will result in an expensive repair and shutdown of power production. Prevention, or at least early notification, of impact-induced wear is essential for preventing economic loss and enhancing personnel safety. Another example of mechanical impacting is the reed valve system used in small refrigeration compressors. During the suction and release process, valve reed will repeatedly impact the valve seat. Impact stresses have been identified as the most common cause of valve failures [10]. A similar situation also happens for heart valves, which slam shut with each heartbeat. In fact, structures known as "contactors" are common in numerous electromechanical components, including motors and relays. In explosive impacts, the damage is often localized and visible with external inspection, which will receive high attention. While under low velocity impact, the impactor has longer contact time with the target. Therefore, the deformation of structure will be global with normally invisible and

${ }^{*}$ Corresponding author: Elizabeth K. Ervin, Civil Engineering, The University of Mississippi, Box 1848, University, MS, 38677 USA. Tel.: +1662915 5618; Fax: +1662915 5523; E-mail: eke@ olemiss.edu. 
hard detectable damage even far from the contact point. Even under normal service loading, the internal damage may grow rapidly to failure. This will dramatically decrease the structural stiffness and service life. Comprehensive phenomenological studies are needed for reducing impact-induced damage. Researchers from multiple fields are working toward this goal from several viewpoints. The relevant research base can be divided into the areas of discrete models, continuous models, modeling techniques, and laboratory experiments.

Structural response under impact has been examined through discrete models. Using both experimental and numerical methods, Vorst et al. [21] concluded that a multi-degree of freedom model is essential to reach a full representation of beam impact. Khalili et al. [13] studied the transient response of a composite panel under low-velocity transverse impact by using a discrete three-degree-of-freedom system. Despite the lack of resolution, contact location was found to be a vital parameter that influences structural response. Discrete models can simplify impact mechanics, but some dynamic behavior may be neglected. Therefore, some researchers have switched to continuous models. The level of contact modeling however has not reached that of discrete systems. Chen et al. [5] studied the problem of a moving rigid body transversely impacting an unrestrained Timoshenko beam. A generalized Fourier series method was performed to obtain the analytical solution of this system. The mass ratio of beam to rigid body was an important parameter in their analysis. Using the lumped parameter method, Lin and He [15] studied the transverse impact problems on simply supported beams and rectangular plates. Impact force and structural deflection are the two parameters they have analyzed. In order to avoid plastic deformation, a trial and error procedure was applied in advance to obtain the maximum impact velocity: this demonstrates the effort required to calibrate such models. By applying the implicit Euler method and the midpoint rule, Ahn et al. [1] studied the system of an Euler-Bernoulli beam with frictionless contact, confirming energy conservation. Chun et al. [6] provided a numerical solution for a point mass impacting a uniform orthotropic beam. Lagrange's Principle and a nonlinear contact law were employed to derive analytical solutions, and the authors concluded that boundary condition was an important factor for the contact force, contact time, stress condition and beam displacement. These studies demonstrate that interesting input and output parameters have been identified and that model complexity can be built into materials or methods.

With so many models for such a common occurrence, experimental studies have also been undertaken. The most applicable studies are those that follow herein. An impact load cell was introduced by Wagg et al. [22] to monitor the dynamic response of a cantilever beam. A probability density method was also mentioned to reconstruct structural parameters by employing the collected data. A pinned beam excited at a comparatively low frequency was tested and results were evaluated with that of both one- and multi-degree of freedom models by Fegelman and Grosh [11]. A brass beam and a hemi-cylindrical aluminum stop were employed in their experiment. Along with accelerometers, a single-point laser vibrometer system was applied to measure the velocity at the contact point. The test was designed to respond significantly at high frequencies (up to $10 \mathrm{kHz}$ ). The author concluded that multi-degree of freedoms are necessary to predict high frequency response. A singly constrained cantilever beam with and without tip mass were studied by Shaw [20] in both experimental and theoretical ways. The thickness of the beam was comparatively small as 0.23 millimeters versus 188 millimeters in length. The specimen was periodically excited though an electromagnetic shaker. A strain gage was applied to measure the motion at one particular position on the specimen. The author stated that the single mode model can give qualitative information on practical mechanisms. Wagg and Bishop [23] applied the non-smooth dynamics approach to a cantilever beam for analyzing the impact phenomenon. Numerical results were compared with the experimental response of a beam/impact system. Sticking motions due to friction were discussed in detail. Experimental and analytical studies were carried out by Adan et al. [2] for the behavior of post-buckling beam under contact constraints. During the test, a constant rate end-shortening displacement was applied through a compression machine on a fixed-roller beam specimen. The transverse deflection at the middle of the specimen was monitored by an electromagnetic LVDT gauge. A simple numerical system has been developed by Bishop et al. [4] to model the dynamic response of a beam-stop structure. During the test, a steel beam was mounted vertically with the free end on top and a rod which screwed into the wall was employed to be the contactor. A sinusoidal force was applied through an electromagnetic transducer, and the response of the beam was captured using a displacement transducer. After comparisons, the author concludes that the model can capture qualitative and, to some extent, quantitative behaviors. A striker and target beam type of experimental setup was used by Masri et al. [17] to verify their numerical model. Strain gages were used to capture the response of the specimen, and the response on the base of the specimen and the shaker were also monitored. The excitation frequency was changed 


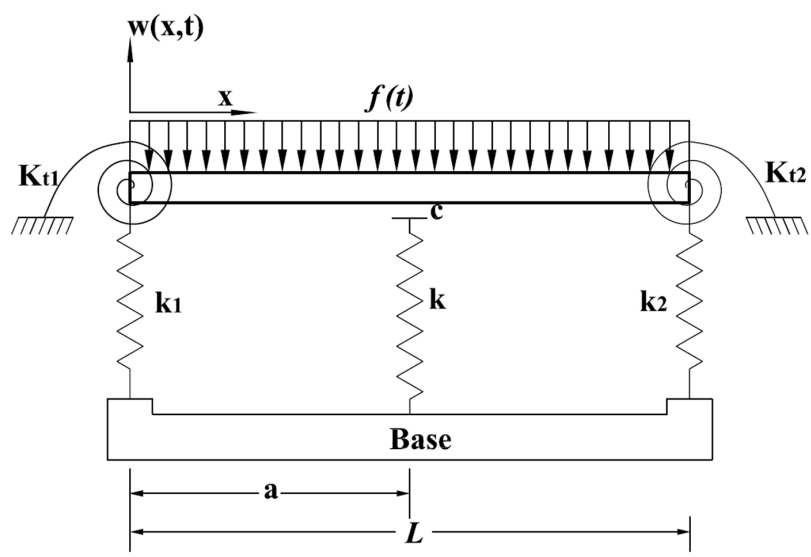

Fig. 1. Beam model with adjustable boundary conditions and variable single-sided contact at location $a$ with gap $c$.

based upon the fundamental frequency of the specimen. The author concluded that maximum deformations are dramatically influenced by the stiffness of specimen and the damping ratio of the striker, but less so by the gap size. Similar structures have been analyzed in such studies as Moon [18], Knudsen [14], and Nagai [19]. Note that results are applicable to repetitive impact which is not induced by transient excitation.

The effects of parametric changes have also been further examined. Chattopadhyay [7] studied the dynamic behavior of a beam-like structure impacting through a clearance. The contact stiffness and the gap magnitude were the only two parameters analyzed in the study, and just the fundamental mode was considered in the analysis. Lin and Bapat [16] joined the describing function, optimization, and spectral analysis approaches to a beam-stop system. Their sensing was far-field, and they examined only impact force and clearance value. Two different experimental systems, an impact beam system and a spatial slider crank apparatus, were studied by Gu et al. [12]. By applying a sinusoidal forcing through an electrical shaker and capturing the impact force using a piezoelectric ceramic contact sensors, the two mechanisms were tested and compared with numerical model. The author mentioned that the impact force is strongly affected by the gap size, excitation frequency, and the dimension of specimen.

Despite significant work in modeling and solution techniques, usually constant or periodic loads are examined; seldom has literature focused on a transient stress pulse. Discrete models are capable of showing harmonic response complexity but are unable to capture the excitation of higher order modes; thus, a continuous model is vital. Direct comparison between experimental and simulation work is also rare when dealing with impact. The nature of this non-linearity often prevents parametric understanding, especially for complex numerical methods which often use only specific cases. With these points in mind, this work will examine the coupled phenomenon of transient beam behavior with impact. Understanding the joint results can help uncover possible ways to prevent impact-induced damage. An analytical model which is discrete in time and continuous in space will be developed for transient beam behavior with impact by applying both modal analysis and time stepping. In contrast to most numerical methods, the analytical solution will allow for quantitative parameterization while simultaneously considering frequency dependency. Unique experiments are also carried out to verify the developed model.

\section{Model}

A generalized Euler-Bernoulli beam with contact location and stiffness will be studied numerically through a model which is discrete in time and continuous in space. The dynamic response of a beam with adjustable boundary and contact conditions is investigated. Illustrated in Fig. 1, the modeled beam has a rectangular cross-section with depth $b$, height $h$, and second moment of area $I$. Other parameters of the beam are the flexural rigidity $E I$, mass density $\rho$, and longitudinal length $L$. Torsional springs $K_{t 1}$ and $K_{t 2}$ and translational springs $k_{1}$ and $k_{2}$ form the adjustable boundaries. The parameter $k$ represents the effective stiffness of the contact interface, and the parameter $a$ identifies the location of the contactor from the left hand side of the beam. The deflection of the beam is denoted as $w(x, t)$. 
The equation of motion for the beam is prescribed by

$$
\rho A w(x, t)_{, t t}+E I w(x, t)_{, x x x x}=f(t)
$$

where comma notation represents differentiation, $A$ is the crossectional area $b h$, and $f(t)$ is the forcing function.

Based upon modal analysis, the deflection is

$$
w(x, t)=\sum_{n=1}^{\infty} \eta_{n}(t) W_{n}(x)
$$

where $W_{n}(x)$ represents the $n$th normalized spatial mode shape and $\eta_{n}(t)$ is the $n$th generalized time coordinate. Note that orthogonality of the modes is required for this formulation.

The separation of variables from Eq. (2) allows Eq. (1) for free vibration to be expressed in each mode as

$$
\begin{aligned}
W_{n}(x)_{, x x x x}-\frac{\rho A \omega_{n}^{2}}{E I} W_{n}(x) & =0 \\
\text { and } \eta_{n}(t)_{, t t}+\omega_{n}^{2} \eta_{n}(t) & =0
\end{aligned}
$$

where $\omega_{n}$ is the $n$th modal natural frequency.

The general solution for Eq. (3) is

$$
W_{n}(x)=C_{1} \cos \left(\beta_{n} x\right)+C_{2} \sin \left(\beta_{n} x\right)+C_{3} \cosh \left(\beta_{n} x\right)+C_{4} \sinh \left(\beta_{n} x\right)
$$

where $C_{1}$ through $C_{4}$ are modal coefficients based upon the boundary conditions and the natural frequencies are $\omega_{n}=\beta_{n}^{2} \sqrt{\frac{E I}{\rho A}}$.

For any $n$, the four boundary conditions in solving for the modal coefficients are as follows:

$$
\begin{aligned}
K_{t 1} W_{n}(0)_{, x} & =E I W_{n}(0)_{, x x} \\
E I W_{n}(0)_{, x x x} & =-k_{1} W_{n}(0) \\
-K_{t 2} W_{n}(L)_{, x} & =E I W_{n}(L)_{, x x} \\
E I W_{n}(L)_{, x x x} & =k_{2} W_{n}(L)
\end{aligned}
$$

When a forcing function is considered, the substitution of Eq. (2) into Eq. (1) results in

$$
\rho A \sum_{n=1}^{\infty} W_{n}(x) \eta_{n}(t)_{, t t}+E I \sum_{n=1}^{\infty} W_{n}(x)_{, x x x x} \eta_{n}(t)=f(t)
$$

Multiplying by an $m$ th mode shape and integrating along beam length, Eq. (6) becomes

$$
\rho A \int_{0}^{L} \sum_{n=1}^{\infty} W_{n}(x) W_{m}(x) \eta_{n}(t)_{, t t} d x+E I \int_{0}^{L} \sum_{n=1}^{\infty} W_{n}(x)_{, x x x x} W_{m}(x) \eta_{n}(t) d x=\int_{0}^{L} f(t) W_{m}(x) d x .
$$

The orthonormality property of

$$
\rho A \int_{0}^{L} W_{n}(x) W_{m}(x) d x=\delta_{n m},
$$

where $\delta_{n m}$ is the Kronecker delta, prescribed that all $m \neq n$ terms are zero. Equation (7) can now be separated into

$$
W_{n}(x)_{, x x x x}-\frac{\rho A \omega_{n}^{2}}{E I} W_{n}(x)=0
$$




$$
\eta_{n}(t)_{, t t}+\omega_{n}^{2} \eta_{n}(t)=\int_{0}^{L} f(t) W_{n}(x) d x=\left\langle f(t), W_{n}(x)\right\rangle
$$

If including damping in the system, Eq. (8) is

$$
\eta_{n}(t)_{, t t}+2 \xi_{n} \eta_{n}(t)_{, t}+\omega_{n}^{2} \eta_{n}(t)=\left\langle f(t), W_{n}(x)\right\rangle
$$

where $\xi_{n}$ is the damping ratio of the $n$th mode. Note that any potential sources of modal damping are bundled as a percentage of velocity loss; this includes material damping. The term $\left\langle f(t), W_{n}(x)\right\rangle$ represents the projection of the force $f(t)$ onto the $n$th mode.

A constant instantaneous force is considered over a time interval $t_{i}$ such that

$$
f(t)=\left\{\begin{array}{lr}
F_{i} & 0<t \leqslant t_{i} \\
0 & t>t_{i}
\end{array}\right.
$$

Any forcing function can be modeled by building up a series of these rectangular pulses. Continuity is maintained by using the end conditions of $i$ th rectangular pulse as the initial conditions for the $(i+1)$ th pulse. Note that small enough time steps are required to capture the true pulse shape.

If the system starts from the rest state, when $0<t \leqslant t_{i}$,

$$
\left\langle f(t), W_{n}(x)\right\rangle=\int_{0}^{L} f(t) W_{n}(x) d x=\int_{0}^{L} F_{i} W_{n}(x) d x=f_{n}
$$

The solution to Eq. (7) is then

$$
\eta_{n}(t)=\frac{-f_{n}}{\omega_{n}^{2}} \exp \left(-\xi_{n} \omega_{n} t\right)\left[\cos \left(\omega_{d n} t\right)+\frac{\xi_{n} \omega_{n}}{\omega_{d n}} \sin \left(\omega_{d n} t\right)\right]+\frac{f_{n}}{\omega_{n}^{2}}
$$

where $\omega_{d n}$ is the $n$th damped natural frequency and equals $\omega_{n} \sqrt{1-\xi_{n}^{2}}$.

The terminal conditions of the previous interval, in this case the displacement $w\left(x, t_{i}\right)$ and the velocity $w, t\left(x, t_{i}\right)$ at time point $t_{i}$, are the initial conditions for the next interval.

When $t>t_{i}$, there is no applied force, so

$$
\left\langle f(t), W_{n}(x)\right\rangle=0 .
$$

However, the temporal solution becomes a more complicated expression due to initial condition enforcement. After simplifying, the general time coordinate for the $n$th mode is

$$
\eta_{n}(t)=\exp \left(-\xi_{n} \omega_{n} t\right)\left[B_{1} \cos \left(\omega_{d n} t\right)+B_{2} \sin \left(\omega_{d n} t\right)\right]
$$

where

$$
\begin{aligned}
& B_{1}=\frac{\eta_{n}\left(t_{i}\right) Q_{, t}-\eta_{n}\left(t_{i}\right)_{, t} Q}{P Q_{, t}-P_{, t} Q} \\
& B_{2}=\frac{\eta_{n}\left(t_{i}\right) P_{, t}-\eta_{n}\left(t_{i}\right)_{, t} P}{Q P_{, t}-Q_{, t} P}
\end{aligned}
$$

and

$$
\begin{aligned}
& P=\exp \left(-\xi_{n} \omega_{n} t\right) \cos \left(\omega_{d n} t\right) \\
& Q=\exp \left(-\xi_{n} \omega_{n} t\right) \sin \left(\omega_{d n} t\right)
\end{aligned}
$$


The motion of the beam structure is divided into two states $T^{f}$ and $T^{c}$ within which vibration is linear and the aforementioned time solutions apply. State $T^{f}$ refers to the "free" condition that the structure is not in contact with the spring; state $T^{c}$ refers to the "contact" situation that the structure is in contact with the deflecting spring. The equation of motion 1 for the beam in state $T^{c}$ is the same as that in the state $T^{f}$, except for the additional compatibilities at the contact location $x=a$ of

$$
\begin{aligned}
& w^{c}\left(a^{-}, t\right)=w^{c}\left(a^{+}, t\right) \\
& w_{, x}^{c}\left(a^{-}, t\right)=w_{, x}^{c}\left(a^{+}, t\right) \\
& E I w_{, x x}^{c}\left(a^{-}, t\right)=E I w_{, x x}^{c}\left(a^{+}, t\right) \\
& E I w_{, x x x}^{c}\left(a^{-}, t\right)-E I w_{, x x x}^{c}\left(a^{+}, t\right)=k w^{c}(a, t) .
\end{aligned}
$$

In order to determine which state of the system plies, the beam deflection at the contact location is monitored. This deflection at any time is calculated via modal analysis as

$$
w(a, t)=\sum_{n=1}^{N} W_{n}(a) \eta_{n}(t)
$$

where $N$ denotes the number of modes in the current state considered in the simulation as discussed in the next section. At relatively small discrete time steps, the deflection at $x=a$ is contrasted with zero; if $w(a, t)>0$, then $T^{f}$ exists, and $T^{c}$ exists when $w(a, t)<0$. When the deflection is zero, the corresponding free or contact modes are used for the calculation in the next time step.

Mapping between the two states is realized through a transformation matrix. This relation is obtained by applying the compatibility conditions at the $j$ th impact or rebound time points, or

$$
\begin{aligned}
& w^{f}\left(x, t_{j}^{-}\right)=w^{c}\left(x, t_{j}^{+}\right) \\
& w_{, t}^{f}\left(x, t_{j}^{-}\right)=w_{, t}^{c}\left(x, t_{j}^{+}\right) .
\end{aligned}
$$

For example, the displacement compatibility through modal analysis from contact to free and vice versa causes

$$
\sum_{p=1}^{m} W_{p}^{f}(x) \eta_{p}^{f}\left(t_{j}^{-}\right)=\sum_{q=1}^{n} W_{q}^{c}(x) \eta_{q}^{c}\left(t_{j}^{+}\right)
$$

where again " $f$ " refers to the free state, " $c$ " refers to the contact state, and $t_{j}$ is the time point when impact occurs.

Premultiplying by $\int_{0}^{L} W_{n}^{c}(x) d x$ and applying the modal orthonormality property,

$$
\eta_{n}^{c}\left(t_{j}^{+}\right)=\sum_{p=1}^{N} \rho A \cdot\left(\int_{0}^{L} W_{p}^{f}(x) W_{n}^{c}(x) d x\right) \cdot \eta_{p}^{f}\left(t_{j}^{-}\right) \quad \text { where } n=1 \text { to } N
$$

Note that the integral term is a constant representing the projection of each state's modes onto the other state, as done by Ervin et al. [8]. This reference also contains proof of modal orthogonality.

Seven influential parameters were previously investigated through numerical case studies [24]. Important factors include structural stiffness as beam height $h$, material as elastic modulus $E$, contact stiffness $k$, contact location $a$, damping ratio $\xi$, pulse duration $t_{1}$, and clearance $c$. For increasing beam height $h$, structural stiffness enlarges, so the cycle time and the maximum deflection decrease. Also, the more flexible the structure is, the more sensitivity to the contact stiffness $k$. Similar trends are shown for increasing elastic modulus $E$. With increasing contact stiffness $k$, the system stiffness increases, the cycle time periods decrease, and less contact time occurs. For higher $k$ values, two or three impacts may exist in each cycle. For any boundary, the contact location $a$ is the parameter that most affects impact and rebound behaviors. Each mode shape directly influenced by the spring location; placing the contactor at a point of maximum deflection causes the greatest rebound response. Increasing the system damping drastically decreases all amplitudes as well as the response complexity. Increasing pulse duration will extend the cycle time and amplify the structure deflection. If the duration is long enough, the beam will vibrate around a balanced position in free motion during the applied forcing. For any boundary condition, increasing gap size will shorten the cycle time and the time portion in the contact state; the deformation amplitude dramatically increases in the contact state. 
Table 1

Test cases for the specimen $(*$ reference case)

\begin{tabular}{lcccc}
\hline Case \# & Amplitude $(\mathrm{g})$ & Duration (millisecond) & Contact location & Gap (millimeter) \\
\hline Case 0 & \multicolumn{1}{c}{ Initial displacement $(2 \mathrm{~mm})$} & $\mathrm{n} / \mathrm{a}$ & $\mathrm{n} / \mathrm{a}$ \\
Case 1 & 9 & 15 & $\mathrm{n} / \mathrm{a}$ & $\mathrm{n} / \mathrm{a}$ \\
Case 2 & 15 & 15 & $0.4840 \mathrm{~L}$ & 0.102 \\
Case $3 *$ & 15 & 15 & $0.4840 \mathrm{~L}$ & 0.229 \\
Case 4 & 15 & 10 & $0.4840 \mathrm{~L}$ & 0.229 \\
Case 5 & 15 & 5 & $0.4840 \mathrm{~L}$ & 0.229 \\
Case 6 & 10 & 15 & $0.4840 \mathrm{~L}$ & 0.229 \\
Case 7 & 5 & 15 & $0.4840 \mathrm{~L}$ & 0.229 \\
Case 8 & 12.5 & 15 & $0.4840 \mathrm{~L}$ & 0.229 \\
Case 9 & 15 & 15 & $0.4840 \mathrm{~L}$ & 0 \\
Case 10 & 15 & 15 & $0.4840 \mathrm{~L}$ & 0.432 \\
Case 11 & 15 & 15 & $0.4840 \mathrm{~L}$ & 0.686 \\
Case 12 & 15 & 15 & $\mathrm{n} / \mathrm{a}$ & $\mathrm{n} / \mathrm{a}$ \\
Case 13 & Initial displacement $(2 \mathrm{~mm})$ & $\mathrm{n} / \mathrm{a}$ & $\mathrm{n} / \mathrm{a}$ \\
\hline
\end{tabular}

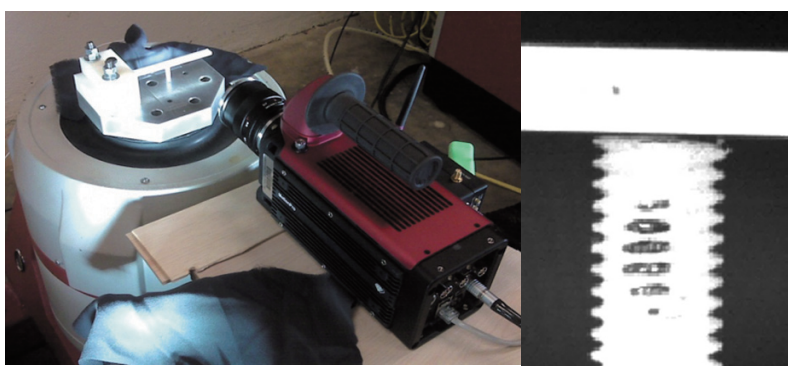

(a)

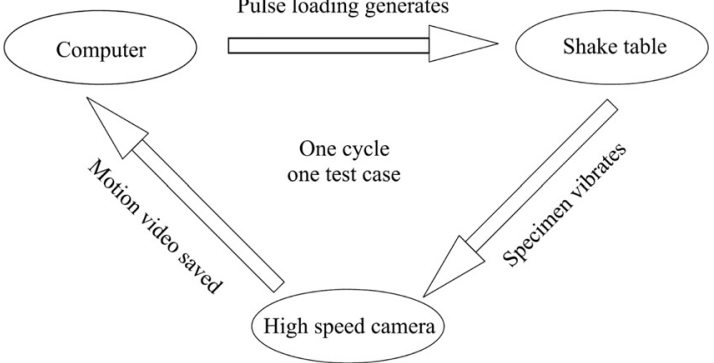

(b)

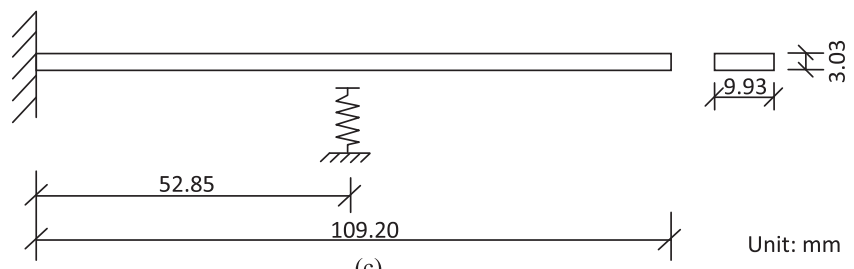

(c)

Fig. 2. (a) Complete test setup and zoom on the contactor. (b) Test procedure. (c) Schematic of the test specimen.

\section{Experiment}

Experiments are used to validate these simulated trend behaviors. With equipment available in the Multi Functional Dynamics Laboratory at the University of Mississippi, an experimental setup was designed as an analog of the numerical model. An LDS 650/PA2000 shaker system in the vertical configuration was used to apply an approximate half-sine pulse loading. The pulse is directed through a DVC vibration controller and a 480-voltage three-phase amplifier. A Redlake Y4 high speed camera with the Motion Studio software package was employed to capture specimen motion. Xcitex's ProAnalyst was then employed to analyze the experimental videos via two-dimensional pattern detection.

A cantilever specimen was designed and fabricated with significant boundary considerations. Three-dimensional models were developed in AutoCAD, and a 3-D printer technique was then employed to form the specimen. The material used is Acrylonitrile Butadiene Styrene (ABS), with an elastic modulus $E$ of $1834 \mathrm{MPa}$ and a density $\rho$ of $951.98 \mathrm{~kg} / \mathrm{m}^{3}$. The length $L$ is 109.20 millimeters, the width $b$ is 9.93 millimeters, and the thickness $h$ is 3.03 millimeters. The dimensions were determined based on the interested frequency range and the shaker adapter plate.

As shown in Fig. 2, the specimen was securely attached to the shaker through two (5/16-inch) threaded steel rods and one (1/4-inch) hex head bolt on the base. For each contact test case, only one contactor was located at $0.4840 \mathrm{~L}$. 
Table 2

Experimental results for three examined parameters

\begin{tabular}{|c|c|c|c|c|c|}
\hline Section \#: Parameter & Parameter value & $\begin{array}{c}\text { Maximum response } \\
\text { (millimeters) }\end{array}$ & $\begin{array}{c}\text { Free duration } \\
\text { (seconds) }\end{array}$ & $\begin{array}{c}\text { Minimum response } \\
\text { (millimeters) }\end{array}$ & $\begin{array}{c}\text { Contact duration } \\
\text { (seconds) }\end{array}$ \\
\hline 3.1: Pulse duration & $\begin{array}{l}15 \text { milliseconds } \\
10 \text { milliseconds } \\
5 \text { milliseconds }\end{array}$ & $\begin{array}{l}1.4554 \\
1.3232 \\
1.1023\end{array}$ & $\begin{array}{l}0.0187 \\
0.0147 \\
0.0122\end{array}$ & $\begin{array}{l}-0.0828 \\
-0.0551 \\
-0.0620\end{array}$ & $\begin{array}{l}0.0040 \\
0.0040 \\
0.0038\end{array}$ \\
\hline 3.2: Pulse amplitude & $\begin{array}{c}10 \mathrm{~g} \\
12.5 \mathrm{~g} \\
15 \mathrm{~g}\end{array}$ & $\begin{array}{l}1.0338 \\
1.2344 \\
1.4554\end{array}$ & $\begin{array}{l}0.0165 \\
0.0194 \\
0.0187\end{array}$ & $\begin{array}{l}-0.1173 \\
-0.0759 \\
-0.0828\end{array}$ & $\begin{array}{l}0.0047 \\
0.0040 \\
0.0040\end{array}$ \\
\hline 3.3: Gap size & $\begin{array}{l}0.000 \text { millimeters } \\
0.229 \text { millimeters } \\
0.432 \text { millimeters }\end{array}$ & $\begin{array}{l}0.2323 \\
1.4580 \\
1.6434\end{array}$ & $\begin{array}{c}\text { Not comparable } \\
0.0187 \\
0.0200\end{array}$ & $\begin{array}{l}\text { Not comparable } \\
-0.0828 \\
-0.0599\end{array}$ & $\begin{array}{c}\text { Not comparable } \\
0.0040 \\
0.0022\end{array}$ \\
\hline
\end{tabular}

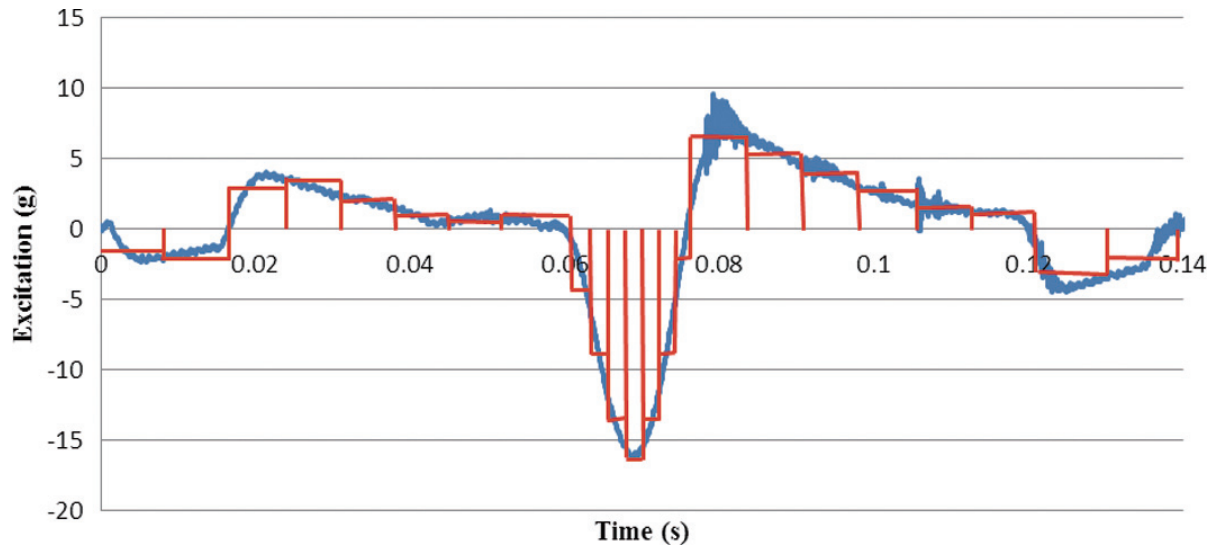

Fig. 3. Discretization of experimental excitation for modeling.

The contactor element was a threaded nylon rod (10/32 inches in diameter) that could be rotated to adjust the gap with the beam.

An initial displacement case is also used to obtain the natural frequencies of the specimen. The beam was deflected from rest and released: the free end displacement was limited to approximately two millimeters to ensure linear behavior. Using the tracked time history, the frequency response is obtained. The first natural frequency of the thick beam is $57.13 \pm 2.20 \mathrm{~Hz}$, and the second natural frequency is $355.96 \pm 2.20 \mathrm{~Hz}$. The natural frequencies were checked at both the beginning and the end of the test series, and no shift or structural damage occurred throughout the tests.

Three parameters were considered: pulse duration, pulse amplitude, and gap size. Thirteen test cases were performed as shown in Table 1; any observed change was based upon a reference case (Case 3) and only one parameter was modified with respect to the reference case for one test case. Test parameters were chosen based upon compatibility with the numerical simulation, and actual test values were obtained in accordance with small deflection theory and shaker capabilities.

As shown in Fig. 3, the input time history was monitored by a 10 millivolts/g table accelerometer; as the shaker is a mechanical apparatus, the amplitude and shape could not be exactly enforced by the controller. The exact trace of the shake table acceleration must be modeled for practical numerical simulation. Output pulses of shaker were compared for repeatability and varied less than $0.2 \%$. The discretized constant force steps that are employed for modeling are also shown.

All numerical results are provided in Table 2, and analysis is provided herein.

\subsection{Pulse duration}

For peak amplitude of $15 \mathrm{~g}$ 's, single half-sine pulses with various durations are applied as shown in Fig. 4(a). Cases 3, 4, and 5 correspond to durations of 15, 10, and 5 milliseconds each; note the inability of the shaker system 


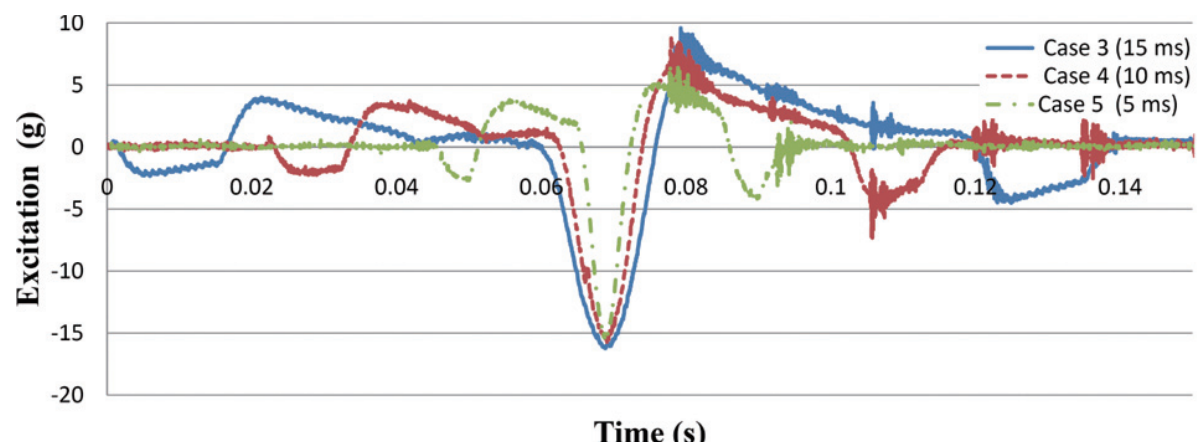

Time (s)

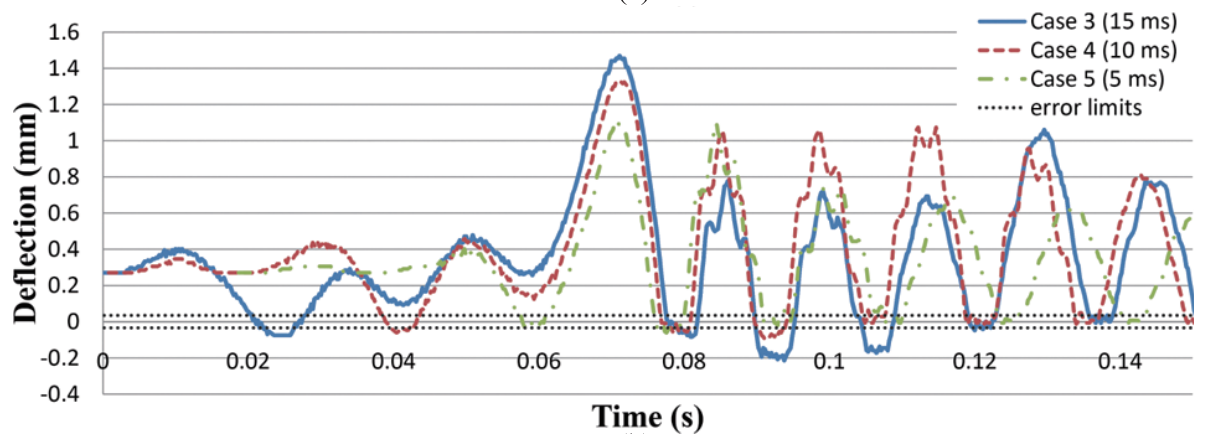

(b)

Fig. 4. (a) Excitation and (b) response when changing pulse duration.

to provide the ideal pulse. Processed experimental results are provided in Fig. 4(b) for the contact point $0.4840 L$ as measured from the fixed boundary via video analysis.

A deflection less than zero indicate that the structure enters into a contact state. When shortening the excitation pulse, the maximum free response decreases as shown in Fig. 2. This makes sense because there is less input energy for the shorter pulse. When the pulse duration is 15 milliseconds, the maximum response is 1.4554 millimeters; by decreasing the pulse duration to 10 and then 5 milliseconds, the maximum deflection lowers $9.08 \%$ and $24.26 \%$, respectively. The duration that the cantilever in the free state lowers $21.39 \%$ when decreasing the pulse duration by $33 \%$. If the pulse duration is further lowered by $66 \%$, the time in the free state will be $34.76 \%$ shorter. Decreasing the pulse duration will shorten the time of beam in the free state as well.

When the specimen first contacts after the peak deflection, minimum responses are provided in Table 2. By decreasing the pulse duration of $33 \%$ and then $66 \%$, the absolute minimum deflection lowers $33.44 \%$ and $25.15 \%$, respectively. Thus, the absolute minimum deflection in the contact state does not have a linear relationship with respect to pulse duration. Decreasing the duration of pulse does not significantly influence the time in the contact state.

\subsection{Pulse amplitude}

Maintaining a 15 millisecond pulse duration, increasing the pulse amplitude will enlarge the deflection as shown in Fig. 5. When the pulse peak value is $10 \mathrm{~g}$ (Case 6), the maximum deflection is 1.0338 millimeters as shown in Table 2. If the pulse is $25 \%$ stronger, the deflection will increase $19.41 \%$; if $50 \%$ stronger, the deflection will further increase $40.79 \%$. The rebound behavior around 0.13 seconds is due to the cool-down of the shaker controller. Cases 6,8 , and 3 show that the time in the free state increases $17.58 \%$ if the pulse is $25 \%$ stronger; if $50 \%$ stronger, the time in the free state increases $13.33 \%$. Therefore, the time in the free state does not linearly depend on the pulse amplitude.

When the specimen first enters into the contact state after the peak deflection, the absolute minimum deflection decreases $35.28 \%$ when the pulse is $25 \%$ stronger; if $50 \%$ stronger, the minimum will decreases $29.44 \%$. Increasing 


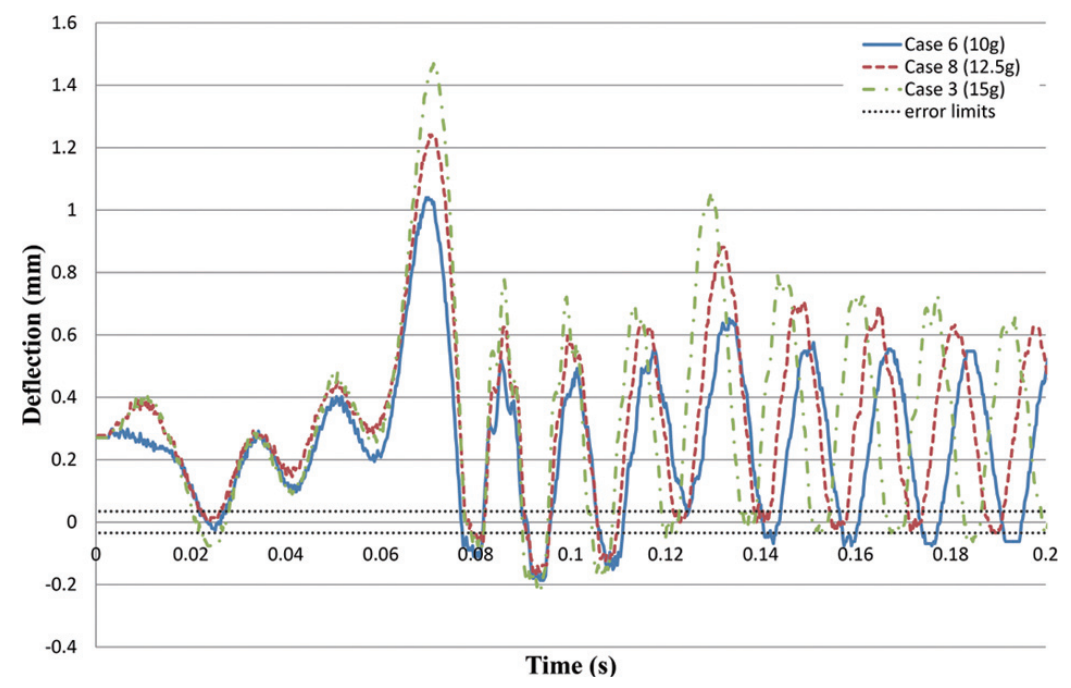

Fig. 5. Response with changing pulse amplitude.

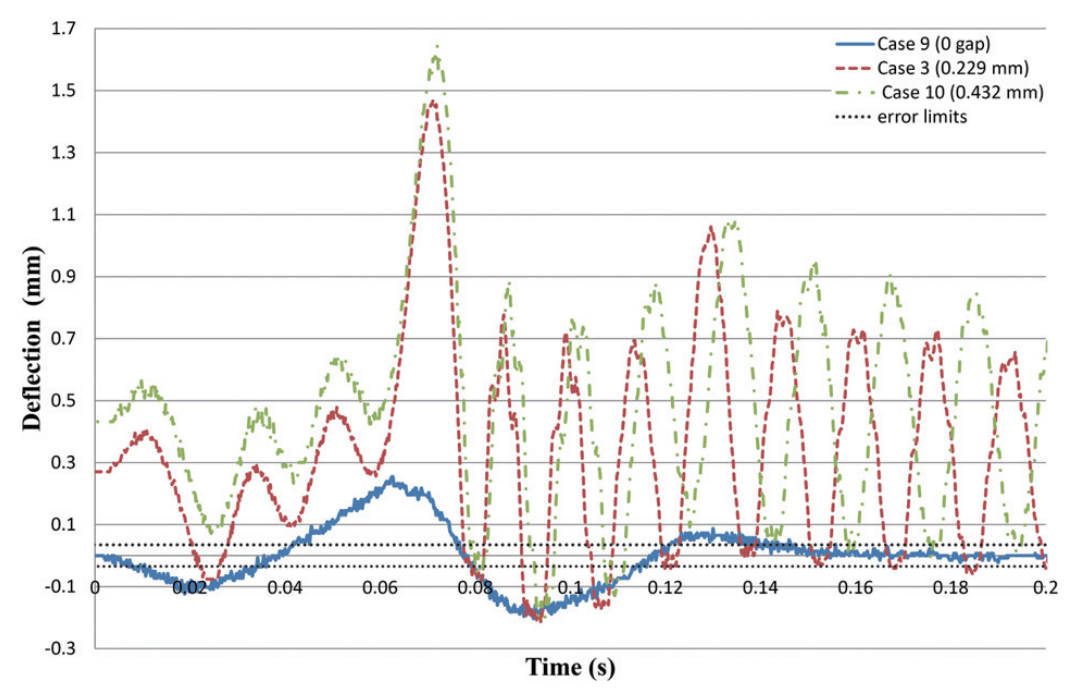

Fig. 6. Comparison of changing gap size.

the pulse amplitude will shorten the time in the contact state; with $25 \%$, and $50 \%$ stronger pulse, the time both decreases $14.89 \%$.

\subsection{Gap size}

Gap size causes a significant change in the experimental time histories. As in Ervin [9], increasing the gap size will enlarge the maximum deflection as shown in Fig. 6. The case with no gap shows the smallest response: this makes sense because the contactor acts more like a stop when there is no gap. When the gap size is 0.229 millimeters (Case 3), the maximum deflection jumps to 1.4580 millimeters due to the increased rebound effect. When the gap size increases $88.65 \%$, the maximum deflection will enlarge further $12.72 \%$. Therefore, contact will augment the structural response under pulse loading, so clearances in machines or structures require high attention. As omitted from Table 2, the time in the free state for the no gap case is not comparable to Case 3 or 10. The latter cases show increasing the gap size will extend the duration in the free state as well. No trend behaviors are identifiable for minimum response or contact duration. 


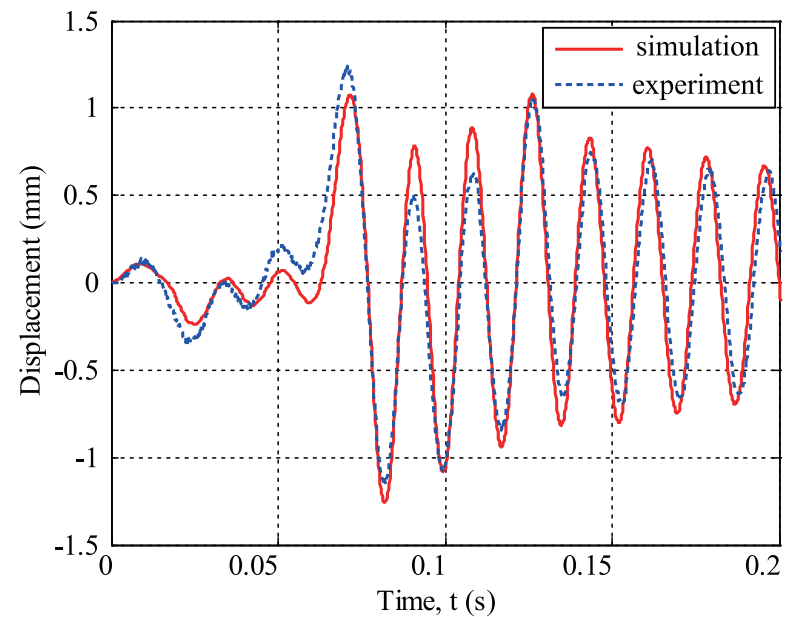

Fig. 7. Comparison of experimental and numerical results for Case 12.

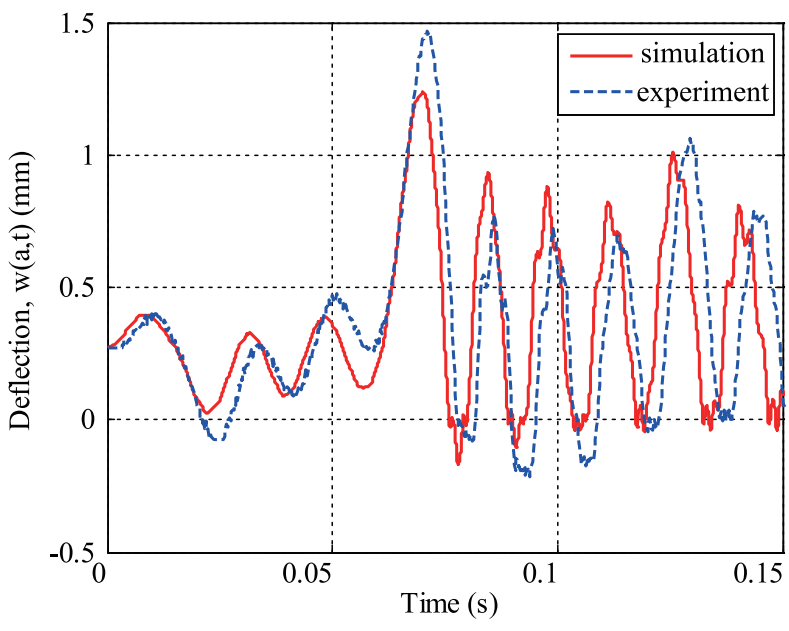

Fig. 8. Comparison of experimental and raw numerical results for Case 3.

\section{Simulation performance}

The comparison of experimental and simulation responses is detailed in this section. A convergence study is included in addition to comparisons of both without and with a contactor. Sources of disparity are also discussed for each case.

Convergence studies ensure that the applied modal analysis has enough higher mode contribution. The applied experimental base acceleration has been discretized as shown in Fig. 3. For this excitation and no contact, the maximum displacement at the first peak has a $0.01 \%$ difference between the 2 -mode and 8 -mode simulations. Therefore, the fundamental frequency dominates for this load case, and higher frequencies are not active. Considering accuracy and computational efficiency, four modes are used for non-contact studies. However, contact causes the activation of higher modes, and convergence for a gap $0.229 \mathrm{~mm}$ at eight modes.

\subsection{Without contactor}

For the non-contact Case 12, the designed maximum amplitude of the excitation is $15 \mathrm{~g}$ 's with duration of 15 milliseconds. The elastic modulus is a somewhat uncertain parameter when using an experimental specimen. The value given by Quickparts (1834 MPa) for the fabricated specimen must be verified for modeling. For this case, the first two natural frequencies from video analysis via ProAnalyst are $57.13 \mathrm{~Hz}$ and $355.96 \mathrm{~Hz}$. The first two numerically calculated natural frequencies are $57.31 \mathrm{~Hz}$ and $359.13 \mathrm{~Hz}$; the difference between the ProAnalyst and numerical results are $0.3 \%$ and $0.9 \%$ for the two modes. Considering the variation range of the experimental results, the given elastic modulus value is acceptable, and no tuning is required.

The estimated damping ratio is calculated by averaging multiple time history analyses using logarithmic decrement method. For this case, the averaged damping ratio used is $1.1 \pm 0.1 \%$. Both viscous damping and material damping comprise this value. The surface friction is neglected; therefore, no Coulomb damping and interfacial damping is considered.

Employing these parameters, the deflection time histories of both the experimental and model results are shown in Fig. 7. The maximum deflections at the first major peak $(t \approx 0.07 \mathrm{~s})$ are 1.08 and 1.24 millimeters for the simulation and test, respectively. The minimum responses at the first negative peak $(t \approx 0.08 \mathrm{~s})$ are -1.26 and -1.15 millimeters. These values are important for response prediction and show error from $9.57 \%$ to $13.31 \%$. More disparity occurs in the second and third positive peaks, but after that the Fig. shows comparatively good overlay. Thus, the decay of the response is well modeled. For duration of the response, 0.01012 seconds and 0.01052 seconds are the time period in the first negative peak for numerical and experiment, and the difference is $3.8 \%$. Therefore, better temporal prediction is reached by the model than that of the amplitude with raw parameters. 


\subsection{With contactor}

When comparing the numerical result to experimental cases with contactor, the contact stiffness is an uncertain factor. In this research, the $k$ value was obtained by matching the fundamental frequency of the specimen in the contact state. This is different with reference by Antunes [3], from which the contact stiffness was decided by the measured impact force. To determine its frequency content, an FFT was performed on the contact portion of a captured response. In order to force identical contact natural frequencies, the contact stiffness needs to be $4030 \mathrm{~N} / \mathrm{m}$.

The contact reference case Case 3 is used for direct comparison. The designed maximum amplitude of the excitation force is $15 \mathrm{~g}$ with duration of 15 milliseconds. The contact is at $0.4840 \mathrm{~L}$ from the fixed boundary with a clearance of 0.229 millimeters. Because of the dissipation of energy by the contactor, the damping ratio of the system should be greater while in contact. The averaged damping ratio of $1.1 \%$ in the free state and $2 \%$ in the contact state was utilized in the simulation.

As shown in Fig. 8, the maximum deflections at the first major peak $(t \approx 0.075 \mathrm{~s})$ are 1.237 and 1.469 millimeters for the simulation and experiment, with a difference of $15.79 \%$. The minimum deflections for the first contact state $(t \approx 0.08 \mathrm{~s})$ are -0.1703 and -0.08275 millimeters for the simulation and experiment. The duration for the first contact state is 0.00397 and 0.004 seconds for the simulation and experiment; the difference is $0.75 \%$. Therefore, as in the case without a contactor, the model can provide better prediction of duration than amplitude. Note that the rebound response $(t \approx 0.13 \mathrm{~s})$ is identified.

As the contact stiffness directly affects the response, underestimation of this value may be the reason why the amplitude difference at the first contact part has been dramatically enlarged. Another possible reason would be the aforementioned underestimation of the excitation force; this explains why the first major peak is undervalued. Further study by increasing the pulse loading and contact stiffness value confirmed this presumption. With $k$ being $7000 \mathrm{~N} / \mathrm{m}$ and increasing the pulse by $20 \%$, the amplitude difference is about $6 \%$ for free state and $17 \%$ for contact state.

\section{Conclusions}

Prevention, or at least early notification, of impact-induced wear is essential for preventing economic loss and enhancing personnel safety. Thus, an efficient model which is discrete in time and continuous in space was undertaken; an Euler-Bernoulli beam with adjustable boundary conditions and variable impact is numerically studied under a pulse loading. This model is limited to global structural effects; a higher order beam model is suggested for local contact stress estimation. Structural stiffness, material modulus, contact stiffness, contact location, damping ratio, pulse duration, clearance and boundary conditions are considered.

Experiments were carried out to verify the effects of these influential parameters. A half-sine pulse excitation was applied through a mechanical shaker, and the deflection was captured by a high speed camera. Numerous test cases on a cantilever beam were conducted that varied pulse duration, pulse amplitude, and clearance. Decreasing the pulse duration lowers all deflection amplitudes, but the time in contact is insensitive. No gap causes the smallest beam response, and increasing clearance generates greater free deflection amplitude.

Representative test cases were then selected for validating the theoretical model. When comparing numerical simulation with experimental result for both specimens, satisfactory agreement for amplitude and duration can be reached even with raw input parameters of the cases without contactor. When there is a contactor, the model shows reasonable results. Contact stiffness and pulse amplitude are two possible sources of error for the difference in comparison.

The piecewise linear contact technique herein is applicable to more complex models. A natural extension of this work is to include advanced constitutive models. Employing variable moduli will provide a more realistic model of damaged structures. Adding directional components will allow for the examination of composites as well. The incorporation of shear stress, such as in a Timoshenko beam, will better represent crack development and local fatigue. 


\section{References}

[1] J. Ahn and D.E. Stewart, An Euler-Bernoulli beam with dynamic contact: Discretizations, convergence and numerical results, Journal of Numerical Analysis 43(4) (2005), 1455-1480.

[2] N. Adan, I. Sheinman and E. Altus, Post-buckling behavior of beams under contact constraints, Journal of Applied Mechanics 61(4) (1994),764-772.

[3] J. Antunes, F. Axisa and M.A. Vento, Experiments on vibro-impact dynamics under fluid elastic instability, Pressure Vessels and Piping Division 189 (1990), 127-138.

[4] S.R. Bishop, M.G. Thompson and S. Foale, Prediction of period-1 impacts in a driven beam, Proceedings of the Royal Society of London, Series A: Mathematical, Physical and Engineering Sciences 452(1954) (1996), 2579-2592.

[5] R. Chen, H. Zheng and S. Xue, Analysis on transverse impact response of an unrestrained Timoshenko beam, Applied Mathematics and Mechanics 25(10) (2004), 1304-1313.

[6] L. Chun and K.Y. Lam, Behavior of uniform anisotropic beams of rectangular section under transverse impact of a mass, Journal of Shock and Vibration 4(2) (1997), 125-141.

[7] S. Chattopadhyay, Dynamics of vibrating beams impacting around a clearance gap, Proceedings of the International Modal Analysis Conference-IMAC 1 (2001), 593-599.

[8] E.K. Ervin and J.A. Wickert, Repetitive impact response of a beam structure subjected to harmonic base excitation, Journal of Sound and Vibration 307(1-2) (2007), 2-19.

[9] E.K. Ervin and J.A. Wickert, Experiments on a beam-rigid body structure repetitively impacting a rod, Nonlinear Dynamics 50(3) (2007), 701-716.

[10] A. Futakawa and J. Nomura, Impact fatigue behavior of flapper valve steel, Proceedings of 1982 Purdue Compressor Technology Conference (1982), 119-126.

[11] K.J.L. Fegelman and K. Grosh, Dynamics of a flexible beam contacting a linear spring at low frequency excitation: Experimental and analysis, Journal of Vibration and Acoustics 124(2) (2002), 237-249.

[12] P. Gu, S. Dubowasky and C. Mavroidis, The design implications of chaotic and near-chaotic vibrations in machines, Proceedings of the 1998 ASME Design Technical Conferences (1998).

[13] M.R. Khalili, K. Malekzadeh and R.K. Mittal, Effect of physical and geometrical parameters on transverse low-velocity impact response of sandwich panels with a transversely flexible core, Composite Structures 77 (2007), 430-443.

[14] J. Knudsen and A.R. Massih, Dynamic stability of weakly damped oscillators with elastic impacts and wears, Journal of Sound and Vibration 263 (2003), 175-204.

[15] Z. Lin and Z. He, Analysis of elastic impact problems on simply supported beams and plates, Journal of the Chinese Society of Mechanical Engineers 8(4) (1987), 231-237.

[16] S.Q. Lin and C.N. Bapat, Extension of clearance and impact force estimation approaches to a beam-stop system, Journal of Sound and Vibration 163(3) (1993), 423-428.

[17] S.F. Masri, Y.A. Mariamy and J.C. Anderson, Dynamic response of a beam with a geometric nonlinearity, Journal of Applied Mechanics 48(2) (1981), 404-410.

[18] F. Moon and S.W. Shaw, Chaotic vibrations of a beam with non-linear boundary conditions, Journal of Nonlinear Mechanics 18(6) (1983), $465-477$.

[19] K. Nagai, S. Maruyama and K. Sakaimoto, Experiments on chaotic vibrations of a post-buckled beam with an axial elastic constraint, Journal of Sound and Vibration 304 (2007), 541-555.

[20] S.W. Shaw, Forced vibrations of a beam with one-sided amplitude constraint: Theory and experiment, Journal of Sound and Vibration 99(2) (1985), 199-212.

[21] E.L.B. van de Vorst, M.F. Heertjes and D.H. van Capen, Experimental and numerical analysis of the steady state behavior of a beam system with impact, Journal of Sound and Vibration 212(2) (1998), 321-336.

[22] D.J. Wagg, G. Karpodinis and S.R. Bishop, An experimental study of the impulse response of a vibro-impacting cantilever beam, Journal of Sound and Vibration 228(2) (1999), 243-264.

[23] D.J. Wagg and S.R. Bishop, Application of non-smoothing modeling techniques to the dynamics of a flexible impacting beam, Journal of Sound and Vibration 256(5) (2002), 803-820.

[24] W. Xu and E.K. Ervin, Analysis of transient beam behavior with impact, ASCE Journal of Engineering Mechanics 137(11) (2011), 779784 

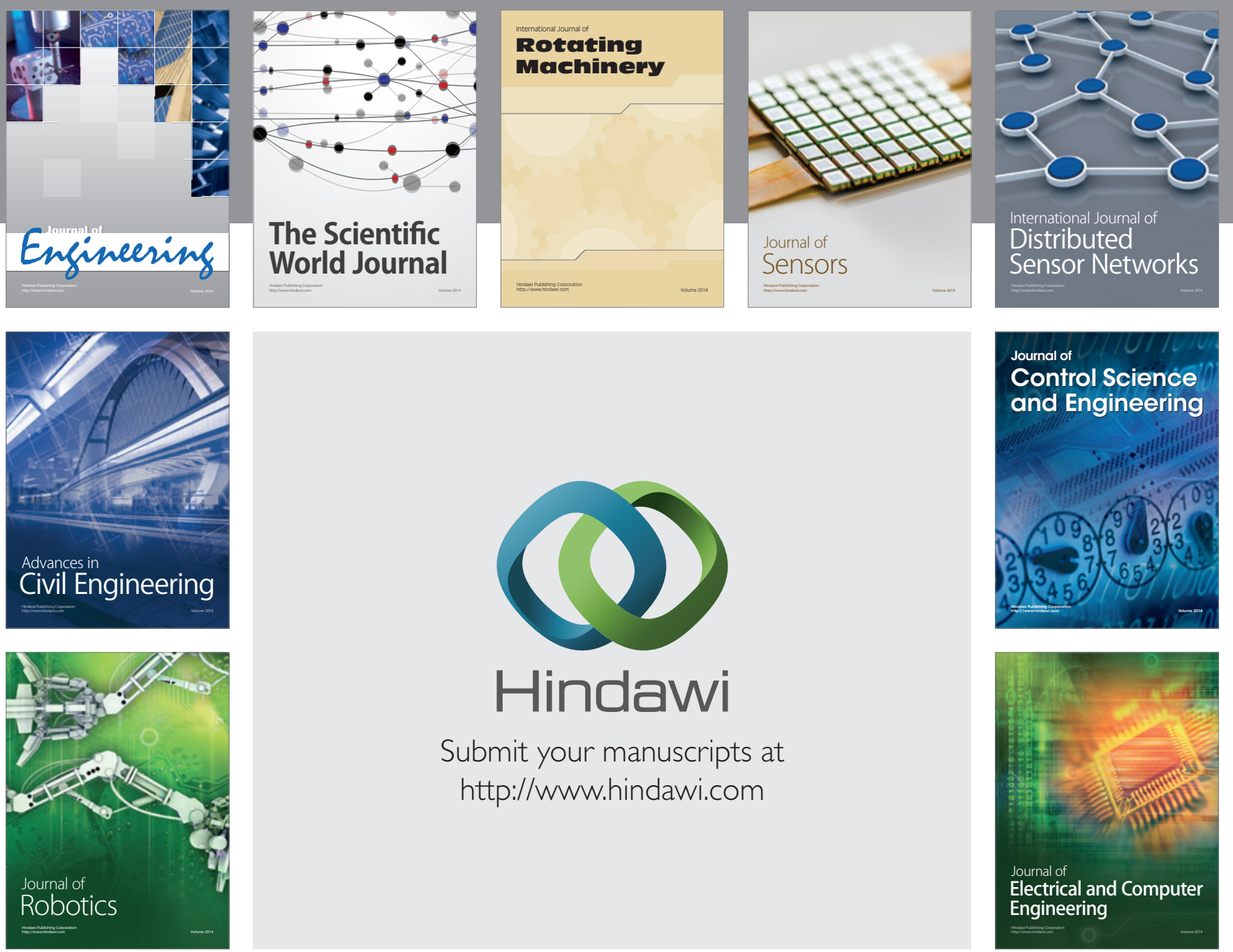

Submit your manuscripts at

http://www.hindawi.com
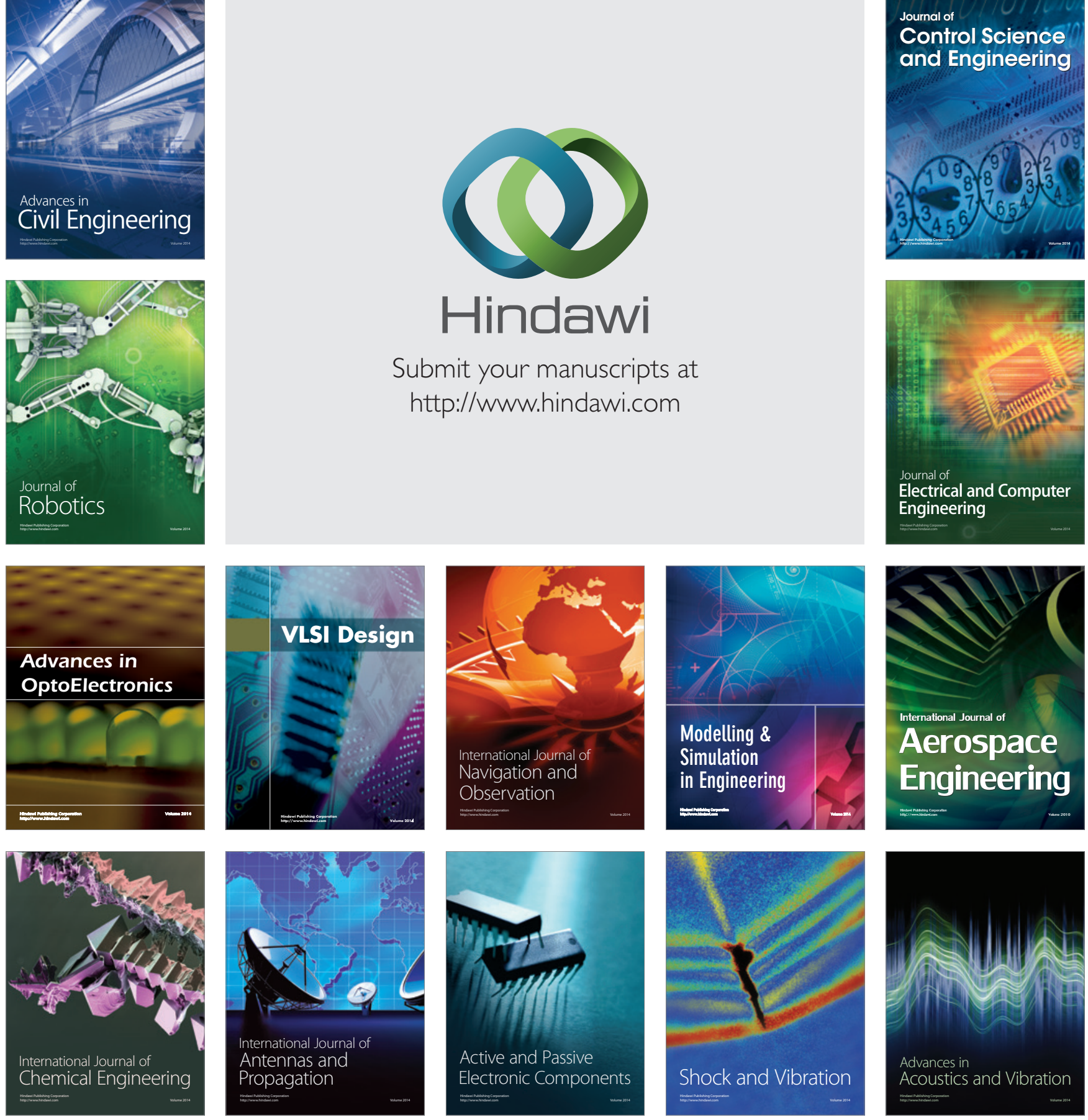